\title{
Molecular epidemiology of hepatitis C infections in Ningxia, China: genotype, phylogeny and mutation analysis
}

\author{
Zhonglan $\mathrm{Wu}^{1 \dagger}{ }^{1}$, Lijia Cui ${ }^{2 \dagger}$, Weiming Zhao ${ }^{3}$, Dongzhi Yang ${ }^{1}$, Hui Chen ${ }^{1}$, Ruiqing Wang ${ }^{4}$, Xuemin Wang ${ }^{1}$,
} Linqi Zhang ${ }^{5}$ and Tianhua $\mathrm{He}^{2^{*}}$

\begin{abstract}
Background: Current prevalence and genotype distribution of hepatitis $\mathrm{C}$ virus (HCV) infection remain unknown in Ningxia, northwest China.

Methods: From June to December 2013, 13,022 individuals were screened in Ningxia HIV/AIDS Sentinel Surveillance System, with their demographic features collected and serum samples tested for HCV antibody. Sero-positive drug users were further subjected to sequencing of NS5B and Core regions of HCV.

Results: The anti-HCV prevalence was $0.34 \%$ among individuals without history of drug use, while it was $15.80 \%$ among drug users. Of 79 NS5B sequences amplified from drug users, 64 (81.0\%) were male and 51 (64.0\%) were injection drug users (IDUs). Subtype $3 \mathrm{a}(40.5 \%)$ and $1 \mathrm{~b}(25.3 \%)$ were the most predominant subtypes, followed in frequency by $3 \mathrm{~b}(10.1 \%)$ and $2 \mathrm{a}(7.6 \%)$. Subtype distribution has no significant difference between injection and non-injection drug users. Based on phylogeographic analysis, HCV strains in Ningxia IDUs were mainly originated from two sites, Yunnan province (in southwest China bordering Myanmar, also known as Burma) and Xinjiang Autonomous Region (in northwest China on the border of Central Asia), which are the two major drug trafficking originates in China. Previously reported drug-resistance mutations were also scanned in this treatment-naïve population. Amino acid substitutions (C316N) associated with direct anti-viral agents (DAA) resistance were identified in the NS5B region in seven samples.
\end{abstract}

Conclusion: This study is the first to reveal the existence of multiple genotypes of HCV in Ningxia, an inland province in northwest China, suggesting the rapid spreading of the virus.

Keywords: Hepatitis C virus, Prevalence, Genotype, Phylogenetic, Genetic diversity

\section{Background}

Hepatitis $\mathrm{C}$ is the second leading cause of chronic hepatitis, liver cirrhosis and hepatocellular carcinoma (HCC) in China. The seroprevalence of hepatitis $\mathrm{C}$ infection in China is about $0.43 \%$ in general population, corresponding to 5.6 million people [1]. However, it has been estimated that the real seroprevalence of $\mathrm{HCV}$ may be close to 10 million in China [2]. In recent years, although the overall hepatitis $\mathrm{C}$ virus $(\mathrm{HCV})$ prevalence is decreasing due to mandatory $\mathrm{HCV}$ antibody screening

\footnotetext{
* Correspondence: hth09@mails.tsinghua.edu.cn

${ }^{\dagger}$ Equal contributors

${ }^{2}$ Tsinghua University School of Medicine, Beijing 100084, China

Full list of author information is available at the end of the article
}

prior to blood transfusion [3-5], the prevalence among populations with high-risk behaviors remain high, especially among injection drug users (IDUs) [6, 7]. Studies have shown that the prevalence of hepatitis $\mathrm{C}$ among IDUs is $70 \%$ in China [6, 8], compared to that of $60 \%$ in the United States [9].

$\mathrm{HCV}$, a member of the family Flaviviridae, is a positive single-stranded RNA virus. HCV is mainly divided into 7 genotypes and more than 80 subtypes. HCV genotype 1 , 2 , and 3 are commonly distributed around the world [10]. Several nationwide studies have shown that genotype 1, 2, 3, 6 were the most prevalent genotypes in China $[11,12]$. The current genetic diversity of HCV infection remains unclear in Ningxia Hui Autonomous 
Region, which is known for its ethnic diversity with about 64.58 \% Han, $35.42 \%$ Hui (Muslims) and less than $1 \%$ other minorities (Fig. 1) [13]. Han is the dominant ethnic group in China with $90 \%$ of the population, while most of the Hui ethnic minority inhabit in Ningxia, is descended from the Arabic and Persian merchants who came to China during the 7th century [14].

As HIV and HCV share the same routes of transmission, we recruited patients from Ningxia HIV/AIDS Sentinel Surveillance System, part of the nationwide disease management program in China launched in 1995 [15, 16]. The HIV/AIDS Sentinel Surveillance System is under the direction of the Ministry of Health $(\mathrm{MOH})$ and the Chinese Center of Disease Control and Prevention (CDC), with nine key affecting populations (KAPs) were screened for HIV infections and related behaviors (Additional file 1: Supplementary Information 1). HCV testing was added and routinely performed since 2009 [17]. Blood samples, collected from KAPs registered from June to December 2013, were subjected to HCV tests, gene sequencing of NS5B, Core regions and further analysis.
HCV genotyping and phylogenetic analysis provide opportunity to reveal the recent $\mathrm{HCV}$ epidemic in Ningxia and the possible transmission networks in China. Such epidemiological and molecular information can also inform the efficacy of current regimens with Peginterferion and Ribavirin, and the upcoming highly effective direct anti-viral agents (DAAs) as well. Besides, few drug-resistance mutations have been reported in China, where no DAAs have not been approved yet. Therefore, pre-treatment testing for individuals may benefit in regimen choosing more effective regimens in the new DAA era.

\section{Methods}

\section{Samples}

Serum samples were collected from Ningxia HIV/AIDS Sentinel Surveillance System, where nine groups of key affecting populations (KAPs) were tested for HCV routinely, including 1) drug users (IDU and non-injection drug users), 2) blood donors, 3) female sex workers (FSW), 4) men who have sex with men (MSM), 5) sexual

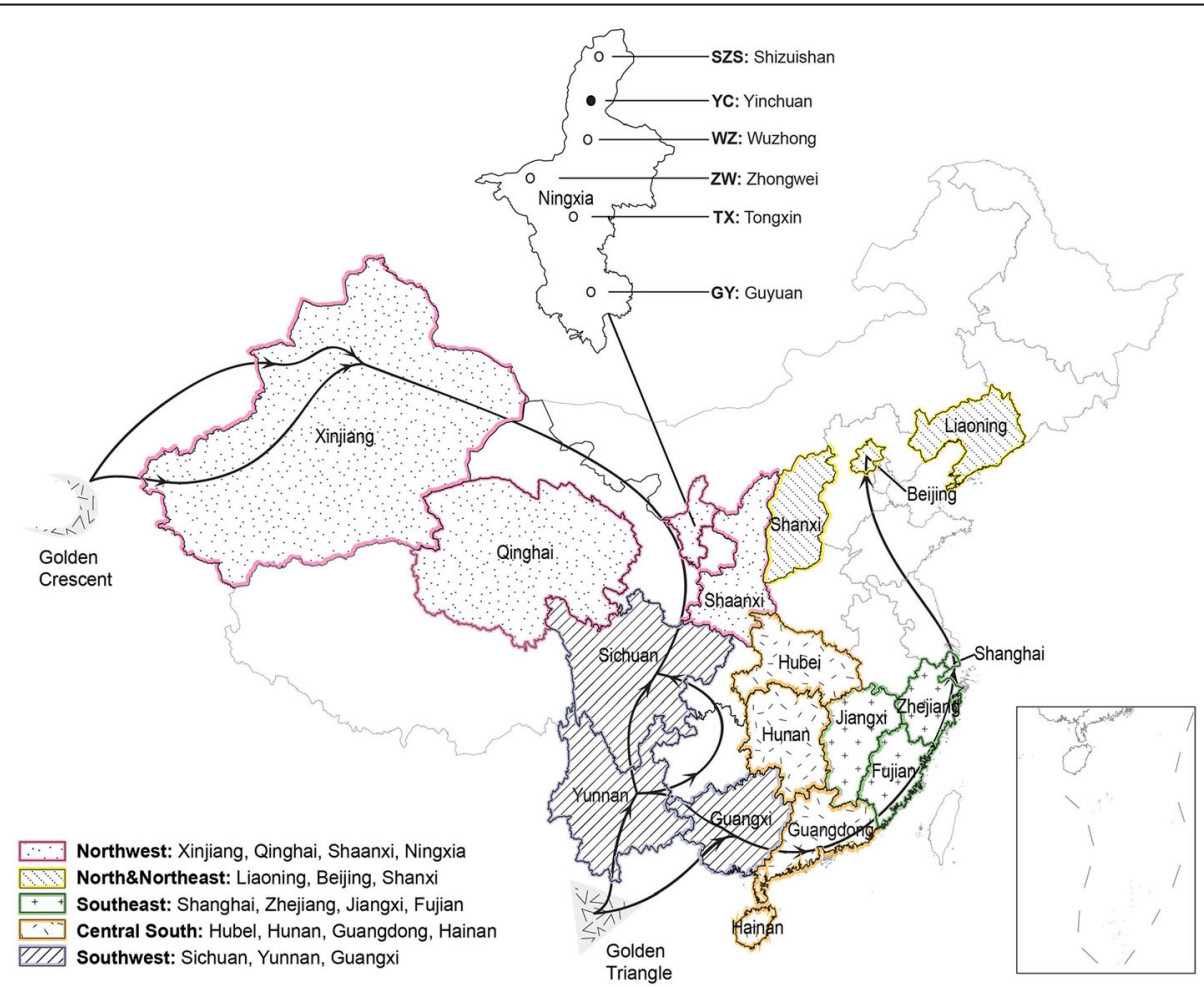

Fig. 1 The map showing sampling spots in Ningxia and 17 provinces or municipalities in China, from which HCV reference sequences were included in the phylogenetic analysis. The 17 provinces and municipalities were divided into five regions and colored accordingly: the northwest (Xinjiang, Qinghai, Shaanxi), north \& northeast (Liaoning, Beijing, Shanxi), southeast (Shanghai, Zhejiang, Jiangxi, Fujian), central south (Hubei, Hunan, Guangdong, Hainan), and southwest (Sichuan, Yunnan, Guangxi). Ningxia is located in northwest China, with six cities Shizuishan (SZS), Yinchuan (YC), Wuzhong (WZ), Zhongwei (ZW), Tongxin (TX), and Guyuan (GY) scattered from north to south where participants were recruited. Major drug trafficking routes were shown by grey arrows referring to Sullivan et al. 2007, which originated from Yunnan (bordering Myanmar and Laos) and Xinjiang (bordering Afghanistan and Pakistan), respectively [39] 
transmitted disease (STD) outpatients, 6) long-distance truck drivers, 7) migratory populations, 8) pregnant women and new mothers, and 9) young students, for HIV, HCV and syphilis screening (Additional file 1: Supplementary Information 1). In this study, only $\mathrm{HCV}$-antibody positive individuals were further informed for detailed characteristics including age, ethnics, occupation, education and drug use history, using custom questionnaires.

\section{HCV antibody and RNA testing, and RNA sequencing}

$\mathrm{HCV}$ antibody testing was conducted using enzymelinked immunosorbant assay (ELISA) with HCV-IgG ELISA kit (Wantai, Beijing, China), and positive results were repeated twice for accuracy. HCV RNA was quantified by real-time PCR, using Roche light cycle 2.0 Diagnostic Kit for hepatitis $\mathrm{C}$ virus RNA (PCR-Fluorescence Probing) (Da'an, Shenzhen, China). HCV RNA positive was defined as RNA copies over $0.5 \times 10^{3} \mathrm{IU} / \mathrm{ml}$. Samples with enough leftovers were further sent for nested PCR and sequencing. Nested PCR was performed as follows: the first round RT-PCR was performed using One-Step RT-PCR kit (TransGene Biotech, Beijing, China) using NS5B and Core specific external primers with the following protocol: $45{ }^{\circ} \mathrm{C} 30 \mathrm{~min}, 94{ }^{\circ} \mathrm{C} 2 \mathrm{~min}$, $\left(94{ }^{\circ} \mathrm{C} 30\right.$ s, $50{ }^{\circ} \mathrm{C} 30$ s, $\left.72{ }^{\circ} \mathrm{C} 90 \mathrm{~s}\right) \times 35$ cycles, and $72{ }^{\circ} \mathrm{C}$ $10 \mathrm{~min}$; the second round PCR was performed using Promega Taq PCR Mastermix kit (Promega Company, USA), using 5 ul product from the first round as the template, and NS5B and Core specific internal primers (Additional file 1: Supplementary Information 3 and Table S2), with the following protocol: $\left(94{ }^{\circ} \mathrm{C} 2 \mathrm{~min}\right.$, $\left.58{ }^{\circ} \mathrm{C} 30 \mathrm{~s}, 72{ }^{\circ} \mathrm{C} \mathrm{90s}\right) \times 30$ cycles, and $72{ }^{\circ} \mathrm{C} 10 \mathrm{~min}$. The final PCR product was tested in agar gel electrophoresis and sent for Sanger sequencing.

\section{HCV genotyping and mutation analysis}

Four hundred fifteen NS5B reference sequences of subtype 1a, 1b, 2a, 2b, 3a, 3b, 6a, 6b, 6n, 6u, 6v were downloaded from GenBank, of which accession numbers were KF585503 - KF585907, GQ206087, EU256071, AB661379, JQ745651, KC844048, EU408332, EU408331, EU408330, D84262, D37855. We performed sequence comparison using the software MEGA 6.0, with the method of Clustal W1.8, and constructed phylogenetic trees using maximum-likelihood (ML) method and GTR $+\mathrm{I}+\Gamma$ model [17-19]. The robustness of reconstructed phylogenies was evaluated by bootstrapping (500 bootstrap replications) to determine genotypes and calculate genetic distances between sequences. The tree topology based on NS5B and Core regions were displayed as a circular form combined with a pie chart of genotype distribution (Fig. 2a, Additional file 1: Figure S1). Of all NS5B sequence alignments, we scanned for previously published DAAresistant mutations on NS5B.

\section{Phylogeographic analysis}

Bayesian coalescent analysis was performed in BEAST software (v1.8.3). The GTR+I+gamma substitution model, uncorrected exponential clock model and Bayesian skygrid coalescent model were selected [20], and $5.0^{*} 10^{-4}$ substitution/site*year was set as the prior rate [21]. The XML file was generated by BEUTi program and ran in BEAST software, with MCMC run for $300,000,000$ states and sampled every 10,000 states. The consensus tree was generated in TreeAnnotator program and Tracer program (v1.6.0), and deciphered in FigTree program (v1.4.2).

\section{Statistical analysis}

We used R 3.0.2 for statistical analysis [22]. HCV subtype distribution was analyzed by Fisher's exact test, and $p$-value less than 0.05 were considered as statistically significant. Kappa values were calculated to estimate the agreement of NS5B and Core genotyping methods (Additional file 1: Supplementary Information 2, and Table S1) [23, 24].

\section{Results \\ Epidemiology}

Among 13,022 individuals recruited from Ningxia HIV/ AIDS Sentinel Surveillance System screened for antiHCV antibody, 2443 were drug users. The overall seroprevalence was $0.34 \%$ among people with no history of drug use, but as high as $5.8 \%$ among drug users. 158 anti-HCV positive specimens were further tested for HCV RNA with 132 being RNA positive. The estimated HCV-RNA positive prevalence was $13.7 \%$ for drug users (Table 1). The prevalence of HCV was significantly higher in IDUs, compared to non-injection drug users.

\section{Demographic features}

Among 79 drug users with a complete set of lab tests and genotype determined, $81.0 \%$ of currently $\mathrm{HCV}$ infected patients were male, $49.4 \%$ were young adults (younger than 40 years old), $87.3 \%$ had middle school or less education, and $69.6 \%$ were unemployed. All drug users have a history of drug use longer than one year, and $64.6 \%$ of them were IDUs. Nine patients (11.4\%), all IDUs, were also HIV co-infected. Detailed demographic characteristics and drug use information were summarized in Table 2.

\section{Genotyping determination}

Seventy-nine of those 116 RNA positive drug users were sent for sequencing for NS5B region of $\mathrm{HCV}$, and nine subtypes were identified in total. Subtype 3a was the 


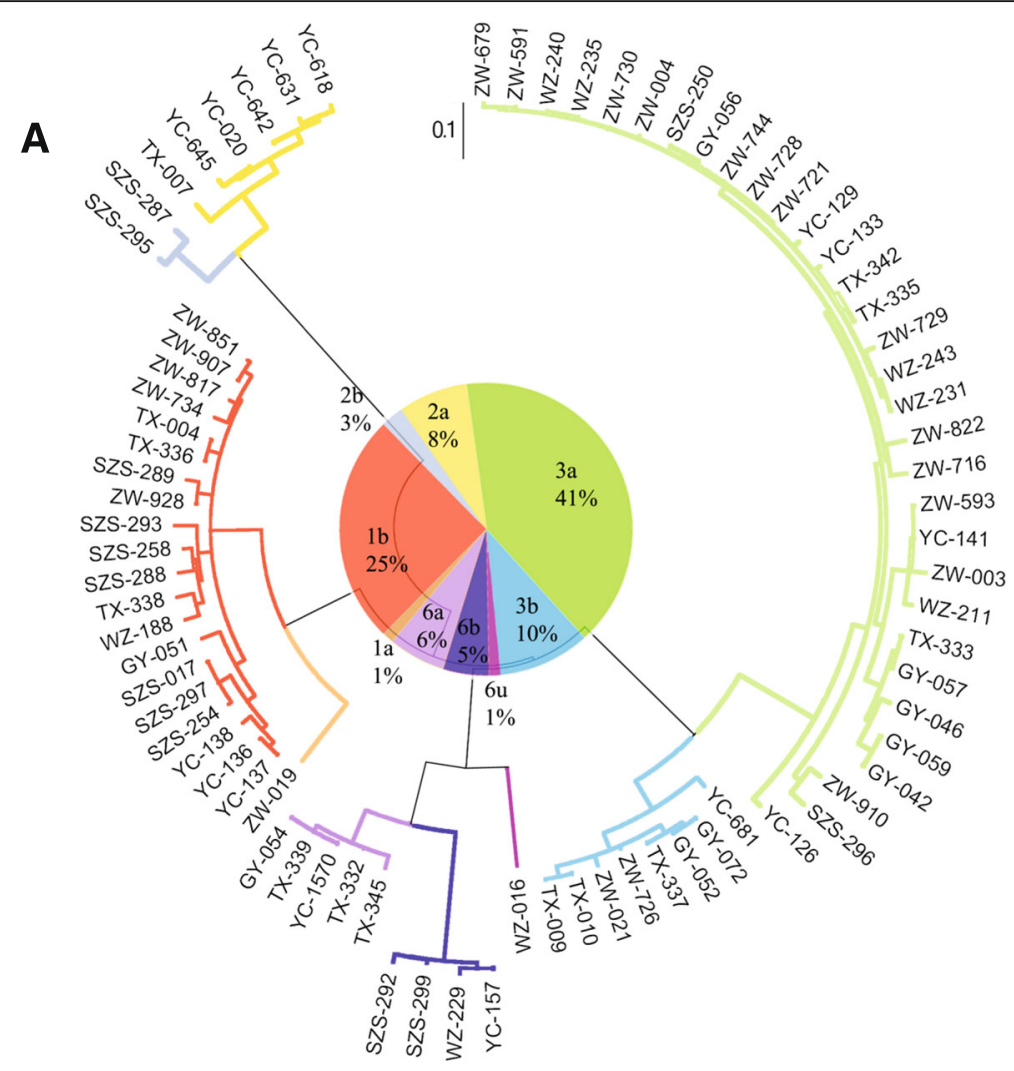

B

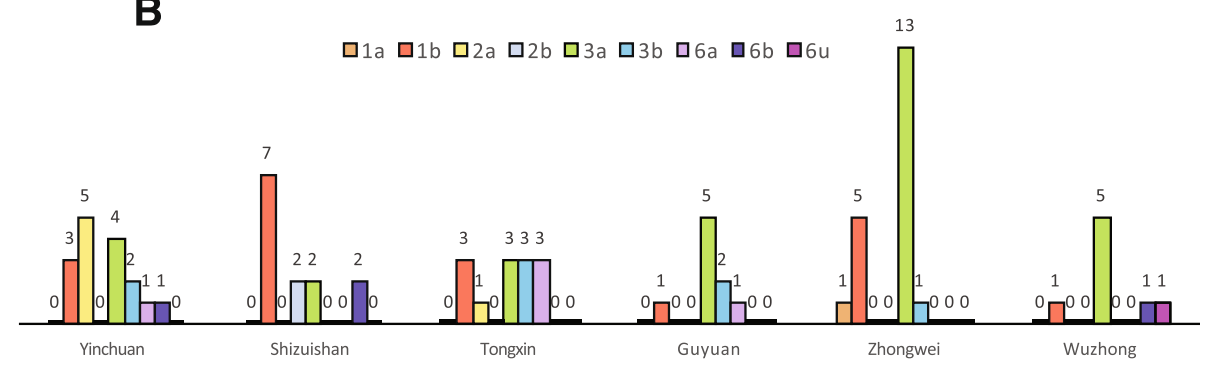

Fig. 2 Subtype distribution of HCV based on the NS5B sequences from 79 subjects. a The circular phylogenetic tree was generated using the software MEGA 6.0, with maximum-likelihood (ML) method and GTR $+1+\Gamma$ model with 500 bootstrap replications. HCV sequences were named by "city of serum collection" and "ID number", where YC, SZS, TX, GY, ZW, and WZ standing for six cities in Ningxia, that is, Yinchuan, Shizuishan, Tongxin, Guyuan, Zhongwei, and Wuzhong, respectively. A pie chart showing the subtype distribution was combined with the tree topology. b A bar chart showing the subtype distribution by cities in Ningxia

most common one, accounting for $40.5 \%$ patients (Fig. 2), followed by subtype $1 \mathrm{~b}, 3 \mathrm{~b}$, and $2 \mathrm{a}$, which took 25.3, 10.1 and $7.6 \%$, respectively. Several rare subtypes were also identified, including five isolates identified as subtype $6 \mathrm{a}$, four isolates as subtype $6 \mathrm{~b}$, two isolates as subtype $2 \mathrm{~b}$, one isolate as subtype $1 \mathrm{a}$, and one isolate as subtype $6 \mathrm{u}$. Compared to subtype $1 \mathrm{~b}$, 3a was more often found to be the younger and less often found among the married (Fisher's exact test, $p<0.05$ ), which indicates an emerging prevalence of subtype 3a infection among the high-risk population in Ningxia. In addition, subtype 1a,
$2 \mathrm{~b}, 6 \mathrm{u}$ and $6 \mathrm{~b}$ were exclusively found in IDUs. Genotyping results based on Core region were present in Additional file 1: Supplementary Information 2 and Figure S1.

\section{Phylogenetic analysis}

Bayesian phylogenetic trees by subtypes were reconstructed for NS5B sequences to explore the possible transmission patterns of $\mathrm{HCV}$ infections, based on a large number of references from 17 provinces or municipalities and a few references of subtype $6 \mathrm{u}, 6 \mathrm{~b}, 1 \mathrm{a}$, and $2 \mathrm{~b}$ strains from other provinces or countries outside 
Table 1 HCV seroprevalence and RNA positive prevalence of the screened population by risk behaviors

\begin{tabular}{|c|c|c|c|c|c|c|c|}
\hline Population by risk factors & $\begin{array}{l}\text { Samples tested } \\
\text { antibody }\end{array}$ & $\begin{array}{l}\text { Samples antibody } \\
\text { positive }\end{array}$ & Sero-prevalence & $\begin{array}{l}\text { Samples tested } \\
\text { RNA }\end{array}$ & HCV RNA+ & $\begin{array}{l}\text { Prevalence of HCV } \\
\text { RNA+ }\end{array}$ & $\begin{array}{l}\text { Sequenced for } \\
\text { NS5B region }\end{array}$ \\
\hline Total & 13,022 & 422 & $3.24 \%$ & 158 & 132 & $2.71 \%$ & 86 \\
\hline Drug users & 2443 & 386 & $15.80 \%$ & 134 & 116 & $13.68 \%$ & 79 \\
\hline Injection drug user & 601 & 284 & $47.25 \%$ & 82 & 73 & $42.07 \%$ & 51 \\
\hline Non-injection drug user & 1842 & 102 & $5.54 \%$ & 52 & 43 & $4.58 \%$ & 28 \\
\hline Not Drug users & 10,579 & 36 & $0.34 \%$ & 24 & 16 & $0.23 \%$ & 7 \\
\hline Blood donors & 2099 & 3 & $0.14 \%$ & 0 & 0 & - & 0 \\
\hline Sex workers & 2737 & 17 & $0.62 \%$ & 17 & 11 & $0.40 \%$ & 5 \\
\hline MSMs & 400 & 0 & $0.00 \%$ & 0 & 0 & - & 0 \\
\hline Patients in STD clinics & 2031 & 7 & $0.34 \%$ & 7 & 5 & $0.25 \%$ & 2 \\
\hline Long-distance truck drivers & 100 & 2 & $2.00 \%$ & 0 & 0 & - & 0 \\
\hline Immigrants & 400 & 1 & $0.25 \%$ & 0 & 0 & - & 0 \\
\hline Pregnant women & 2012 & 2 & $0.10 \%$ & 0 & 0 & - & 0 \\
\hline College students & 800 & 4 & $0.50 \%$ & 0 & 0 & - & 0 \\
\hline
\end{tabular}

Nine groups from high- to low-risk populations were screened in Ningxia HIV/AIDS Sentinel Surveillance System, including 1) drug users (injection drug users and non-injection drug users), 2) blood donors, 3) female sex workers (FSW), 4) men who have sex with men (MSM), 5) sexual transmitted disease (STD) outpatients, 6) long-distance truck drivers, 7) migratory populations, 8) pregnant women, and 9) young students. The detailed criteria of the population were in Supplementary information 1. Prevalence of HCV antibody (seroprevalence) and HCV RNA was higher among injection drug users than that among non-injection drug users

China [19] (Fig. 1). For a better display, the Bayesian phylogenetic tree was split into five subtrees representing different subtypes (Figs. 3 and 4).

Subtype $1 \mathrm{~b}$ used to be the most prevalent subtype in China, and strains from different regions were crossly distributed, suggesting simultaneous dissemination. In Fig. 3a, four groups, namely Group A to D, have been designated in previous studies with different geographic distributions features [19]. Most sequences from Ningxia, however, formed a cluster separately from Group A to D, indicating the local spreading of $\mathrm{HCV}$.

Two major groups of 2a were reported: Group A contained sequences mainly from the northwest China, and Group B from various regions in China [19] (Fig. 3b). Five isolates, all collected from one rehabilitation center in Yinchuan, Ningxia, formed a cluster with the posterior probability of 0.99 in Group A. This cluster might descend from a common ancestor in northwest China dated back to year 1990s. In addition, two closely related isolates from Shizuishan, Ningxia were identified as subtype 2b. As subtype $2 \mathrm{~b}$ was rarely reported in China, such isolates might imply possible real-world transmission pairs.

Subtype 3a sequences formed three separated geographic groups (Fig. 3c). Group A contained the majority of $3 a$ isolates in Ningxia and reference sequences from central south China. Group B mainly contained sequences form southwest China. The rest isolates formed Group C, including sequences from neighboring Xinjiang province, in northwest China, which was in consistency to the known epidemic in the local area. Therefore, we hypothesized that subtype 3a was introduced into Ningxia in 1990s simultaneously from three regions mentioned above. Subtype 3b, unlike 3a, showed substantial mixture of originates, and sequences in Ningxia also contributed to the geographic interspersion (Fig. 3d).

The phylogeny of genotype 6 was reconstructed on the basis of nine Ningxia sequences and 55 reference sequences (Fig. 4). Three groups were noted among subtype 6a. We found that isolates of Ningxia were geographically interspersed among central south provinces in China. Therefore, the possible migration trend might arise from central south, spread to southeast and southwest, and then transmit to the northwest China sporadically. In addition, one close set of Ningxia sequences of subtype 6a was found with full posterior probability of 1.00 in Group B, implying a possible transmission network behind. Meanwhile, four Ningxia isolates clustered together were closely related to two 6b strains, D84262 and D37855, reported in Thailand [25].

The homology of every cluster of subtype was as follows: 1 b $95.14 \%$, 2a $96.91 \%$, 2b $92.90 \%$, 3a $95.69 \%$, 3b $96.72 \%$, 6a $98.27 \%$, 6b $98.30 \%$. Highest homology was associated with subtype $6 \mathrm{a}$ and $6 \mathrm{~b}$, which suggested the late introduction of these subtypes to Ningxia; and the lowest homology $(89.68 \%)$ was associated with subtype $2 b$, indicating its long endemic history [26].

\section{Mutation analysis}

We scanned for previously reported DAA-resistant mutations on NS5B, including S282T and C316N/Y in 79 NS5B sequences [27, 28]. No S282T samples were found, and seven $\mathrm{C} 316 \mathrm{~N}$ resistance samples were found in $1 \mathrm{~b}$ subtype sequences, suggesting the natural occurrence of 
Table 2 Genotype distribution of 79 drug users by demographic features

\begin{tabular}{|c|c|c|c|c|c|c|c|c|c|c|c|c|}
\hline Genotype & & All & $1 a$ & $1 b$ & $2 a$ & $2 b$ & $3 a$ & $3 b$ & $6 a$ & $6 b$ & $6 u$ & $p$-value \\
\hline Overall & & 79 & 1 & 20 & 6 & 2 & 32 & 8 & 5 & 4 & 1 & \\
\hline \multirow[t]{3}{*}{ Sex } & & & & & & & & & & & & 0.589 \\
\hline & Male & 64 & 1 & 17 & 6 & 1 & 24 & 6 & 5 & 3 & 1 & \\
\hline & Female & 15 & 0 & 3 & 0 & 1 & 8 & 2 & 0 & 1 & 0 & \\
\hline \multirow[t]{5}{*}{ Age } & & & & & & & & & & & & 0.013 \\
\hline & $<30$ & 14 & 1 & 4 & 2 & 0 & 5 & 1 & 1 & 0 & 0 & 0.843 \\
\hline & $31-40$ & 25 & 0 & 1 & 1 & 0 & 15 & 3 & 2 & 3 & 0 & 0.010 \\
\hline & $41-50$ & 36 & 0 & 15 & 2 & 2 & 11 & 3 & 1 & 1 & 1 & 0.029 \\
\hline & $\geq 50$ & 4 & 0 & 0 & 1 & 0 & 1 & 1 & 1 & 0 & 0 & 0.117 \\
\hline \multirow[t]{4}{*}{ Marriage } & & & & & & & & & & & & 0.003 \\
\hline & Unmarried & 32 & 0 & 5 & 2 & 1 & 19 & 2 & 2 & 1 & 0 & 0.111 \\
\hline & Married & 16 & 1 & 3 & 4 & 0 & 3 & 4 & 0 & 1 & 0 & 0.004 \\
\hline & Divorced or Widow & 31 & 0 & 12 & 0 & 1 & 10 & 2 & 3 & 2 & 1 & 0.035 \\
\hline \multirow[t]{4}{*}{ Ethnics } & & & & & & & & & & & & 0.163 \\
\hline & Han & 40 & 0 & 12 & 2 & 1 & 18 & 1 & 3 & 2 & 1 & 0.153 \\
\hline & Hui & 31 & 0 & 5 & 3 & 1 & 11 & 7 & 2 & 2 & 0 & 0.033 \\
\hline & Others & 8 & 1 & 3 & 1 & 0 & 3 & 0 & 0 & 0 & 0 & 0.721 \\
\hline \multirow[t]{4}{*}{ Education } & & & & & & & & & & & & 0.175 \\
\hline & Elementary school & 35 & 1 & 10 & 5 & 0 & 9 & 3 & 4 & 3 & 0 & 0.035 \\
\hline & Middle school & 34 & 0 & 7 & 1 & 2 & 19 & 3 & 1 & 0 & 1 & 0.158 \\
\hline & High school or college & 10 & 0 & 3 & 0 & 0 & 4 & 2 & 0 & 1 & 0 & 0.754 \\
\hline \multirow[t]{3}{*}{ Occupation } & & & & & & & & & & & & 0.178 \\
\hline & Employed & 24 & 0 & 6 & 3 & 0 & 7 & 5 & 1 & 1 & 1 & \\
\hline & Unemployed & 55 & 1 & 14 & 3 & 2 & 25 & 3 & 4 & 3 & 0 & \\
\hline \multirow[t]{3}{*}{ Drug use method } & & & & & & & & & & & & 0.402 \\
\hline & Injection drug user & 51 & 1 & 13 & 4 & 2 & 19 & 6 & 1 & 4 & 1 & \\
\hline & Non-injection drug user & 28 & 0 & 7 & 2 & 0 & 13 & 2 & 4 & 0 & 0 & \\
\hline \multirow[t]{6}{*}{ Drug use duration } & & & & & & & & & & & & 0.069 \\
\hline & $<1$ year & 0 & 0 & 0 & 0 & 0 & 0 & 0 & 0 & 0 & 0 & 1.000 \\
\hline & $1-2$ years & 12 & 0 & 4 & 0 & 0 & 6 & 2 & 0 & 0 & 0 & 0.735 \\
\hline & $3-4$ years & 25 & 0 & 5 & 2 & 0 & 7 & 4 & 4 & 3 & 0 & 0.072 \\
\hline & $5-10$ years & 23 & 1 & 3 & 4 & 0 & 12 & 2 & 0 & 0 & 1 & 0.060 \\
\hline & $>10$ years & 19 & 0 & 8 & 0 & 2 & 7 & 0 & 1 & 1 & 0 & 0.117 \\
\hline \multirow[t]{3}{*}{ HIV co-infection } & & & & & & & & & & & & 0.061 \\
\hline & Yes & 9 & 1 & 2 & 2 & 0 & 1 & 2 & 0 & 0 & 1 & \\
\hline & No & 70 & 0 & 18 & 4 & 2 & 31 & 6 & 5 & 4 & 0 & \\
\hline
\end{tabular}

HCV subtypes were determined by the sequence NS5B

${ }^{a}$ Rare subtypes including $1 a, 2 b, 6 b$, and $6 u$ were removed from Fisher's exact test. $p<0.05$ were bolded

DAA-resistant mutations in this treatment-naïve population.

\section{Discussion}

We found that seroprevalence of $\mathrm{HCV}$ was $0.34 \%$ among individuals without drug use history and $15.8 \%$ for drug users in Ningxia, based on a screening on individuals from Ningxia HIV/AIDS Sentinel Surveillance System. Ningxia was conventionally considered as a low-risk area, with the seroprevalence about $0.10 \%$ among general population [1, 29]. Specialists and clinicians suggested a higher prevalence since some high-risk populations were not covered in the previous nationwide survey. Therefore, we mainly focused on drug users 


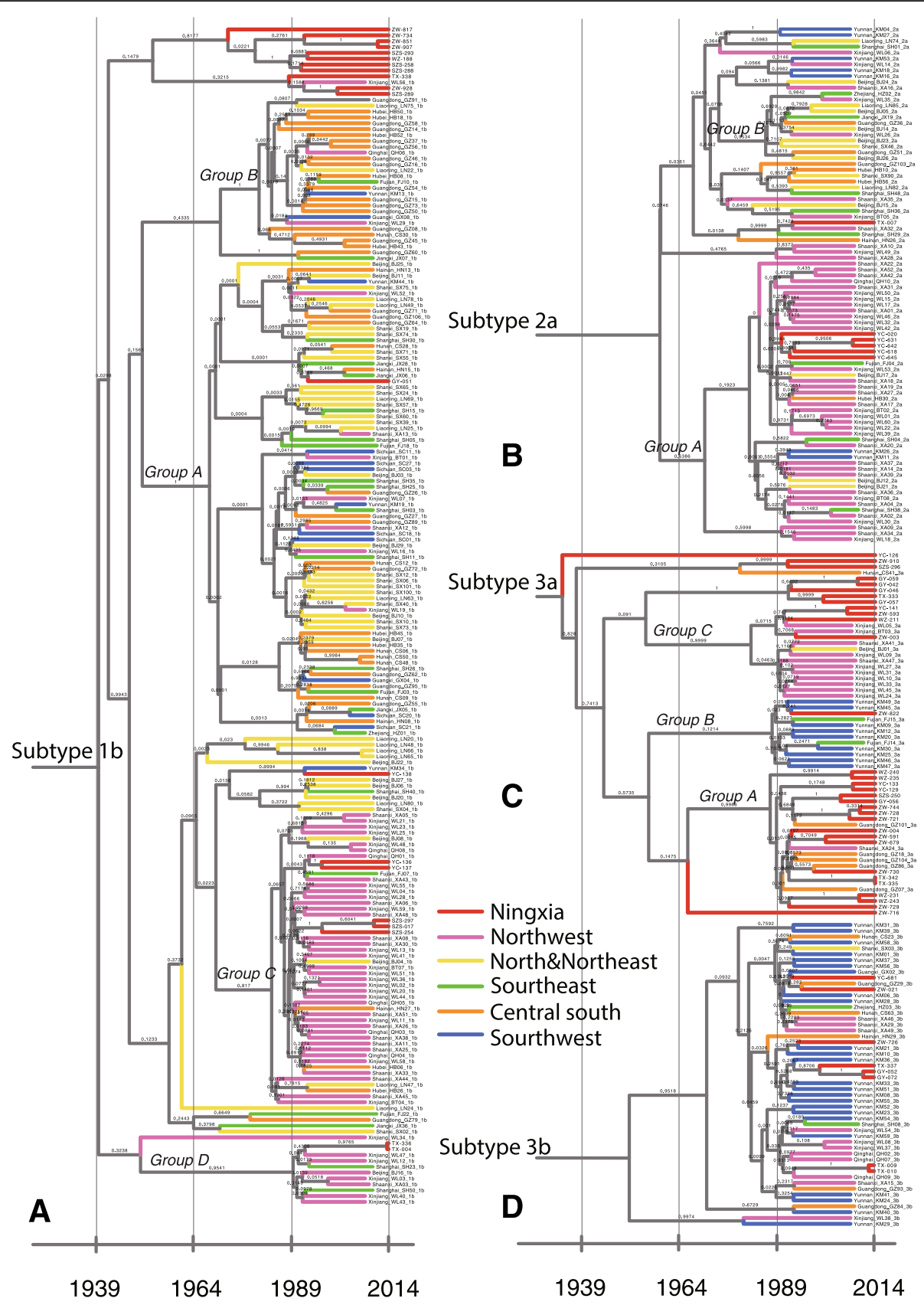

Fig. 3 Bayesian phylogenetic tree based on NS5B from sequences from this study (labeled in red) along with 415 reference strains of $1 b, 2 a$, 3a, and 3b. The geographic location of those sequences was mapped in Fig. 2. a The Bayesian tree of subtype 1b. $\mathbf{b}$ The Bayesian tree of subtype 2a. c The Bayesian tree of subtype 3a. d The Bayesian tree of subtype $3 b$

recruited from HIV surveillance system. We found that the prevalence among drug users was comparable to that of neighboring provinces but lower than the reported prevalence nationwide, that was, $66.97 \%$ for IDUs and $18.97 \%$ for non-injection drug users $[8,30]$.

Previous genotyping studies provided evidences to the hypothesis of two epidemics of HCV in China. Blood transfusion contributed to the first epidemic in China [31]. Subtype $1 \mathrm{~b}$, followed by $2 \mathrm{a}$, was reported to be the most predominant subtypes in those studies that screened more blood donors [19, 32, 33]. Mandatory screening of blood and blood products was implemented in the 1990s to prevent the spreading of $\mathrm{HCV}$ [5]. Together with the increasing number of drug users, several studies have noticed a shift to the second epidemic: from subtype $1 \mathrm{~b}$ to genotype 3 and 6 [34-37]. In addition, IDU is suspected as a factor that drives the emerging of new subtypes mainly due to genetic drift [38]. In our study, nine subtypes were identified, in which 3a 


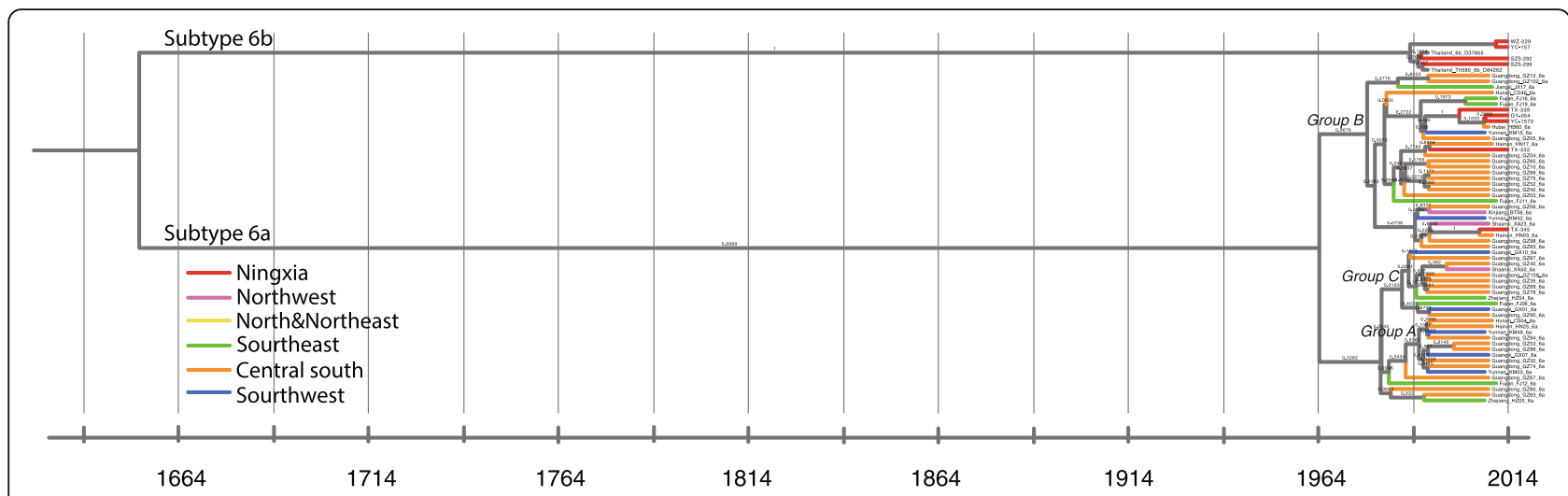

Fig. 4 Bayesian phylogenetic tree based on NS5B from nine sequences (labeled in red) along with 55 references of $6 a$ and $6 \mathrm{~b}$

(41.38 \%) and $1 \mathrm{~b}(25.29 \%)$ were the predominant subtypes. Subtype 1a, 2b, 6u and $6 \mathrm{~b}$ were not reported in $\mathrm{Lu}$ et al. [19], which was the largest genotyping study in China so far. The finding of multiple genotypes in Ningxia including fairly rare subtypes reflected the substantial mixture of HCV patients and accelerated propagation of the disease by modern transportation, internal migration, blood transfusion and IDU.

Studies on drug trafficking routes suggested the correlation of HIV transmission network and the drug smuggling routes from two major heroin-producing regions, "The Golden Triangle" (Myanmar, Laos and Thailand) and "The Golden Crescent" (Afghanistan and Pakistan) [39]. HCV shared the same way of transmission and thus the same epidemic features with HIV. The geographic features of $\mathrm{HCV}$ prevalence reinforced its spreading through these two drug trafficking routes [8]. Ningxia is located at the crossroad of the two drug trafficking routes geographically (Fig. 1). Phylogenetic analysis of subtype 3a also suggested the virus in Ningxia comes from both originates, Yunnan (bordering Myanmar and Laos) and Xinjiang (bordering Afghanistan and Pakistan) (Fig. 3c).

We found 11 co-infected patients of both HIV and HCV (13.9 \%), which is much higher than the prevalence of $1.88 \%$ among drug users in prior studies $[8,40]$. This inconsistency may be due to the sampling bias, as samples collected in this study are from HIV/AIDS Sentinel Surveillance System. Besides, very few studies have been performed on mixed infection of different subtypes of $\mathrm{HCV}$, which may be a hint for viral reconstruction, and a boost to fasten viral evolution [34]. Conventional sequencing methods based on short regions would not enable us to confirm hybrid genetics even if it exists. Further studies need to be performed on the detection of the intra-host viral population with deep sequencing [41].

With regard to the naturally occurring drug-resistance mutations, several single nucleotide polymorphisms (SNPs) of NS5B protease resistance have been previously reported in treatment-naïve hepatitis $C$ patients $[28,42]$.
As the first DAA will be introduced to China soon, searching for drug-resistance SNPs may impact the planning for $\mathrm{HCV}$ treatment and predict the disease burden in the future. S282T was the most common one conferred resistance to NS5 nucleoside analogs inhibitors, such as Sofosbuvir, in subtype $1 \mathrm{a}, 1 \mathrm{~b}$ and modest resistance in subtype 2a $[43,44]$. In this study, we found no such resistance-bearing viral strains. However, C316N were detected in seven isolates among $1 \mathrm{~b}$ sequences, which was reported to be associated with resistance to non-nucleotide inhibitor of NS5 protease [45-47]. Seven C316N-bearing sequences form two fairly close-related clusters, implying a direct transmission network behind, which calls for behavior study to confirm.

The findings of this study may impact the HCV controlling approaches in terms of screening, treatment, and patients management. First, several studies have suggested that interventions targeting drug users were effective approaches for HCV control [48, 49]. Continued screening, monitoring and treatment for drug users may reduce $\mathrm{HCV}$ incidence and prevalence, and could be highly costeffectiveness. Second, needle exchange programs (NEP) and methadone maintenance treatment (MMT) may also be a feasible approach in controlling $\mathrm{HCV}$ transmission among IDUs. There are currently eight NEP/MMT sites operating in Ningxia, but still 23.1 to $44.4 \%$ IDUs reported sharing needles in a recent survey conducted in $2015[50,51]$. Our findings highlighted the necessity of the expansion of NEP in Ningxia and increased financial investment to such programs. In addition, treatment with the implementation of new DAAs could be highly effective, whereas their cost and value had been widely debated as the DAA treatment is much too expensive. Besides, the complex treatment regimen for HCV is further complicated by HIV co-infection $[52,53]$.

This study has several limitations. First, all serum samples were collected from individuals presented in HIV/ AIDS Sentinel Surveillance System in 2013, and therefore the prevalence could not reflect that of general population. 
Further studies on the phylogeny on sequences from general population may present with a different pattern. Second, only NS5B and Core sequences were analyzed in our study. Whole genome sequencing performed on deep sequencing would provide more information on genotype distribution and mixed infection of various strains within one host [41]. Third, we did not collect enough clinical data, such as liver biopsy, aminotransferase/platelet ratio index (APRI) or FIB-4 scores, and thus were not able to stratify our patients by their liver status [54-56].

\section{Conclusion}

In conclusion, the overall seroprevalence of $\mathrm{HCV}$ was $0.34 \%$ among people with no history of drug use in Ningxia, while it was $15.8 \%$ among drug users and $47.25 \%$ among IDUs, based on a screening on individuals from Ningxia HIV/AIDS Sentinel Surveillance System. Nine subtypes, 1a, 1b, 2a, 2b, 3a, 3b, 6a, 6b and $6 \mathrm{u}$ were found among 79 isolates from drug users. 3a (41.38 \%) followed by $1 \mathrm{~b}(25.29 \%)$ were the most predominant. Phylogenetic analysis suggested two possible originates of HCV transmission, accompanying the drug trafficking route in China. Our study suggested that drug use, especial IDU, is the most important risk factor for HCV infection, and management of drug users in Ningxia may play a crucial role in controlling the ongoing HCV epidemic.

\section{Additional file}

Additional file 1: Supplementary information 1. Section 1: Introduction to HIV sentinel surveillance system. Section 2: Inclusion criteria of screened population. Supplementary information 2. Section 1: Sequence of NS5B and Core. Section 2: Measurement of agreement. Table S1: Comparing HCV genotyping results between the Core and NS5B regions. Supplementary information 3. Table S2: NS5B and Core region-specific primers. Supplementary information 4: Figure S1. The circular phylogenetic tree based on the Core sequences. (DOCX $545 \mathrm{~kb}$ )

\section{Abbreviations \\ APRI: Aminotransferase/platelet ratio index; CDC: Chinese Center of Disease Control and Prevention; DAA: Direct anti-viral agents; ELISA: Enzyme-linked immunosorbant assay; FSW: Female sex workers; HCC: Hepatocellular carcinoma; HCV: Hepatitis C virus; IDUs: Injection drug users; KAPs: key affecting populations; ML: Maximum-likelihood; MMT: Methadone maintenance treatment; MOH: Ministry of Health; MSM: Men who have sex with men; NEP: Needle exchange programs; SNPs: Single nucleotide polymorphisms; STD: Sexual transmitted disease}

\section{Acknowledgement}

We thank Dr. Marc A. Suchard from UCLA for the technical support in phylogenetic analysis with BEAST software.

\section{Funding}

This research is supported by grants from Ningxia Science and Technology Program - International Cooperation Program (2013ZYH193) and Ningxia Natural Science Foundation (NZ13217).

\section{Availability of data and material}

One hundred thirty-eight sequences of NS5B and Core regions of hepatitis C virus was submitted to NCBI (the GenBank accession number: KX940824KX940901, KX950601-KX950659).

\section{Authors' contributions}

ZW, study designing and sequencing; LC, phylogenetic analysis and manuscript writing; WZ, study designing; DY, HC, RW, XW, data collecting; LZ, manuscript writing; $\mathrm{TH}$, study designing and manuscript writing. All authors read and approved the final manuscript.

\section{Competing interests}

The authors declare that they have no competing interests.

\section{Consent for publication}

Written informed consents for publication were obtained from all recruited individuals.

\section{Ethics approval and consent to participate}

The Ningxia CDC institutional review board has approved the study. Written informed consents to participate were obtained from all recruited individuals.

\section{Author details}

${ }^{1}$ Ningxia Center for Disease Control and Prevention, Ningxia 750001, China. ${ }^{2}$ Tsinghua University School of Medicine, Beijing 100084, China. ${ }^{3}$ Ningxia Medical University School of Public Health and Management, Ningxia 750001, China. ${ }^{4}$ Wuzhong Center of Disease Control and Prevention, Ningxia 751100, China. ${ }^{5}$ Comprehensive AIDS Research Center, and Collaborative Innovation Center for Diagnosis and Treatment of Infectious Diseases, Tsinghua University School of Medicine, Beijing 100084, China.

Received: 25 July 2016 Accepted: 12 October 2016

Published online: 18 October 2016

\section{References}

1. Chen YS, Li L, Cui FQ, Xing WG, Wang L, Jia ZY, Zhou MG, Gong XH, Wang FZ, Zheng $H$, et al. A sero-epidemiological study on hepatitis C in China. Zhonghua Liu Xing Bing Xue Za Zhi. 2011;32:888-91.

2. Wang FS, Fan JG, Zhang Z, Gao B, Wang HY. The global burden of liver disease: the major impact of China. Hepatology. 2014;60:2099-108.

3. Peng J, Lu Y, Liu W, Zhu Y, Yan X, Xu J, Wang X, Wang Y, Liu W, Sun Z. Genotype distribution and molecular epidemiology of hepatitis $C$ virus in Hubei, Central China. PLoS One. 2015;10:e0137059.

4. Wang J, Liu J, Huang Y, Wright DJ, Li J, Zhou Z, He W, Yang T, Yao F, Zhu X, et al. The persistence of hepatitis $C$ virus transmission risk in China despite serologic screening of blood donations. Transfusion. 2013;53:2489-97.

5. Rao H, Wei L, Lopez-Talavera JC, Shang J, Chen H, Li J, Xie Q, Gao Z, Wang $L$, Wei J, et al. Distribution and clinical correlates of viral and host genotypes in Chinese patients with chronic hepatitis C virus infection. J Gastroenterol Hepatol. 2014;29:545-53.

6. Yuan JM, Govindarajan $\mathrm{S}$, Henderson BE, Yu MC. Low prevalence of hepatitis C infection in hepatocellular carcinoma (HCC) cases and population controls in Guangxi, a hyperendemic region for HCC in the People's Republic of China. Br J Cancer. 1996;74:491-3.

7. Zhang F, Zhu H, Wu Y, Dou Z, Zhang Y, Kleinman N, Bulterys M, Wu Z, Ma Y, Zhao D, et al. HIV, hepatitis B virus, and hepatitis C virus co-infection in patients in the China National Free Antiretroviral Treatment Program, 2010-12: a retrospective observational cohort study. Lancet Infect Dis. 2014;14:1065-72.

8. Bao YP, Liu ZM. Systematic review of HIV and HCV infection among drug users in China. Int J STD AIDS. 2009;20:399-405.

9. Garten RJ, Lai S, Zhang J, Liu W, Chen J, Vlahov D, Yu XF. Rapid transmission of hepatitis $C$ virus among young injecting heroin users in Southern China. Int J Epidemiol. 2004;33:182-8.

10. Gower E, Estes C, Blach S, Razavi-Shearer K, Razavi H. Global epidemiology and genotype distribution of the hepatitis C virus infection. J Hepatol. 2014;61:S45-57.

11. Cui Y, Jia J. Update on epidemiology of hepatitis B and C in China. J Gastroenterol Hepatol. 2013;28 Suppl 1:7-10.

12. Su YY, Liu HX, Wang N. Hepatitis C virus genotypes in China: a systematic review. Zhonghua Liu Xing Bing Xue Za Zhi. 2013;34:80-4.

13. National Bureau of Statistics of China.: Communiqué of the National Bureau of Statistics of People's Republic of China on Major Figures of the 2010 Population Census [1] (No. 2). 29 April 2011.

14. Leslie D. Islam in traditional China: a short history to 1800 . Canberra: Canberra College of Advanced Education; 1986. 
15. Chen X, He JM, Ding LS, Zhang GQ, Zou XB, Zheng J. Prevalence of hepatitis B virus and hepatitis $C$ virus in patients with human immunodeficiency virus infection in Central China. Arch Virol. 2013;158:1889-94.

16. Ministry of Health of China $(\mathrm{MOH})$, National Center for AIDS Prevention and Control (NCAIDS) and Collaboration Group for National HIV Sentinel Surveillance Program. Set up of national sentinel surveillance of HIV infection in China and its report in 1995. Zhongguo Xing Bing Ai Zi Bing Fang Zhi. 1996;2:193-7.

17. Tamura K, Dudley J, Nei M, Kumar S. MEGA4: Molecular Evolutionary Genetics Analysis (MEGA) software version 4.0. Mol Biol Evol. 2007;24:1596-9.

18. Thompson JD, Higgins DG, Gibson TJ. CLUSTAL W: improving the sensitivity of progressive multiple sequence alignment through sequence weighting, position-specific gap penalties and weight matrix choice. Nucleic Acids Res. 1994:22:4673-80

19. Lu L, Wang M, Xia W, Tian L, Xu R, Li C, Wang J, Rong X, Xiong H, Huang K, et al. Migration patterns of hepatitis $C$ virus in China characterized for five major subtypes based on samples from 411 volunteer blood donors from 17 provinces and municipalities. J Virol. 2014;88:7120-9.

20. Gill MS, Lemey P, Faria NR, Rambaut A, Shapiro B, Suchard MA. Improving Bayesian population dynamics inference: a coalescent-based model for multiple loci. Mol Biol Evol. 2013;30:713-24.

21. Purdy MA, Forbi JC, Sue A, Layden JE, Switzer WM, Opare-Sem OK, Phillips $\mathrm{RO}$, Khudyakov YE. A re-evaluation of the origin of hepatitis $C$ virus genotype 2 in West Africa. J Gen Virol. 2015;96:2157-64.

22. R Core Team. R: A language and environment for statistical computing. Vienna: R Foundation for Statistical Computing; 2013. http://www.R-project.org/.

23. Landis JR, Koch GG. The measurement of observer agreement for categorical data. Biometrics. 1977;33:159-74.

24. Nakazawa M: fmsb: Functions for medical statistics book with some demographic data. R package version 0.5.1. http://CRAN.R-project.org/package=fmsb. 2014

25. Tokita H, Okamoto H, Luengrojanakul $P$, Vareesangthip K, Chainuvati T, lizuka H, Tsuda F, Miyakawa Y, Mayumi M. Hepatitis C virus variants from Thailand classifiable into five novel genotypes in the sixth (6b), seventh $(7 \mathrm{c}, 7 \mathrm{~d})$ and ninth (9b, 9c) major genetic groups. J Gen Virol. 1995;76(Pt 9):2329-35.

26. Touzet S, Kraemer L, Colin C, Pradat P, Lanoir D, Bailly F, Coppola RC, Sauleda S, Thursz MR, Tillmann $\mathrm{H}$, et al. Epidemiology of hepatitis $\mathrm{C}$ virus infection in seven European Union countries: a critical analysis of the literature. HENCORE Group. (Hepatitis C European Network for Co-operative Research). Eur J Gastroenterol Hepatol. 2000;12:667-78.

27. Franco S, Casadella M, Noguera-Julian M, Clotet B, Tural C, Paredes R, Martinez MA. No detection of the NS5B S282T mutation in treatment-naive genotype $1 \mathrm{HCV} / \mathrm{HIV}-1$ coinfected patients using deep sequencing. J Clin Virol. 2013;58:726-9.

28. Kuntzen T, Timm J, Berical A, Lennon N, Berlin AM, Young SK, Lee B, Heckerman D, Carlson J, Reyor LL, et al. Naturally occurring dominant resistance mutations to hepatitis $C$ virus protease and polymerase inhibitors in treatment-naive patients. Hepatology. 2008:48:1769-78.

29. Lu J, Zhou Y, Lin X, Jiang Y, Tian R, Zhang Y, Wu J, Zhang F, Zhang Y, Wang Y, Bi S. General epidemiological parameters of viral hepatitis A, B, C, and $E$ in six regions of China: a cross-sectional study in 2007. PLoS One. 2009;4:e8467.

30. Xia X, Luo J, Bai J, Yu R. Epidemiology of hepatitis C virus infection among injection drug users in China: systematic review and meta-analysis. Public Health. 2008;122:990-1003.

31. Zhao SM, Jiang TL, Gao FX, Lu L, Zheng HQ, Hu J, Fan YH, Li B, Xiao RR, Yury K. Analysis of true voluntary blood donors with anti-HCV prevalence and implications for donor management in Chongqing, China. Transfus Med. 2007;17:210-1.

32. Tian D, Li L, Liu Y, Li H, XU X, Li J. Different HCV genotype distributions of HIV infected individuals in Henan and Guangxi, China. PLoS One. 2012;7:e50343.

33. Zhang YY, Lok AS, Chan DT, Widell A. Greater diversity of hepatitis C virus genotypes found in Hong Kong than in mainland China. J Clin Microbiol. 1995;33:2931-4

34. Ye Y, Yan YS, Chen G, Yan PP, Zheng WX, Deng YQ, Yang XH, Wu SL, Zhang ZS. Molecular epidemiology of hepatitis $C$ virus among different groups of people in the province of Fujian, China. Arch Virol. 2013;158:611-8.

35. Xia X, Lu L, Tee KK, Zhao W, Wu J, Yu J, Li X, Lin Y, Mukhtar MM, Hagedorn $\mathrm{CH}$, Takebe $\mathrm{Y}$. The unique HCV genotype distribution and the discovery of a novel subtype $6 \mathrm{u}$ among IDUs co-infected with HIV-1 in Yunnan, China. J Med Virol. 2008;80:1142-52

36. Zhang C, Wu N, Liu J, Ge Q, Huang Y, Ren Q, Feng Q, He G. HCV subtype characterization among injection drug users: implication for a crucial role of Zhenjiang in HCV transmission in China. PLoS One. 2011;6:e16817.
37. Yan Z, Fan K, Wang Y, Fan Y, Tan Z, Deng G. Changing pattern of clinical epidemiology on hepatitis $C$ virus infection in Southwest China. Hepat Mon 2012;12:196-204.

38. Thong VD, Akkarathamrongsin S, Poovorawan K, Tangkijvanich P, Poovorawan Y. Hepatitis C virus genotype 6: virology, epidemiology, genetic variation and clinical implication. World J Gastroenterol. 2014;20: 2927-40.

39. Sullivan SG, Wu Z. Rapid scale up of harm reduction in China. Int J Drug Policy. 2007;18:118-28.

40. Zhuang X, Wang Y, Chow EP, Liang Y, Wilson DP, Zhang L. Risk factors associated with HIV/HCV infection among entrants in methadone maintenance treatment clinics in China: a systematic review and metaanalysis. Drug Alcohol Depend. 2012;126:286-95.

41. Goncalves Rossi LM, Escobar-Gutierrez A, Rahal P. Multiregion deep sequencing of hepatitis C virus: An improved approach for genetic relatedness studies. Infect Genet Evol. 2016;38:138-45.

42. Applegate TL, Gaudieri S, Plauzolles A, Chopra A, Grebely J, Lucas M, Hellard M, Luciani F, Dore GJ, Matthews GV. Naturally occurring dominant drug resistance mutations occur infrequently in the setting of recently acquired hepatitis C. Antivir Ther. 2015;20:199-208.

43. Poveda E, Wyles DL, Mena A, Pedreira JD, Castro-Iglesias A, Cachay E. Update on hepatitis $C$ virus resistance to direct-acting antiviral agents Antiviral Res. 2014;108:181-91.

44. Ji H, Kozak RA, Biondi MJ, Pilon R, Vallee D, Liang BB, La D, Kim J, Van Domselaar $G$, Leonard $L$, et al. Next generation sequencing of the hepatitis C virus NS5B gene reveals potential novel S282 drug resistance mutations. Virology. 2015;477:1-9.

45. Sarrazin C, Zeuzem S. Resistance to direct antiviral agents in patients with hepatitis C virus infection. Gastroenterology. 2010;138:447-62.

46. Trevino A, de Mendoza C, Parra P, Rodriguez C, Madejon A, Plaza Z, del Romero J, Poveda E, Soriano V. Natural polymorphisms associated with resistance to new antivirals against HCV in newly diagnosed HIV-HCVcoinfected patients. Antivir Ther. 2011:16:413-6.

47. Ali S, Leveque V, Le Pogam S, Ma H, Philipp F, Inocencio N, Smith M, Alker A, Kang $\mathrm{H}$, Najera I, et al. Selected replicon variants with low-level in vitro resistance to the hepatitis C virus NS5B polymerase inhibitor PSI-6130 lack cross-resistance with R1479. Antimicrob Agents Chemother. 2008;52:4356-69.

48. Zhang L, Yap L, Xun Z, Wu Z, Wilson DP. Needle and syringe programs in Yunnan, China yield health and financial return. BMC Public Health. 2011:11:250.

49. Jarlais DD. Systematic review research on needle/syringe programs and opiate substitution programs in low- and middle-income countries. J Food Drug Anal. 2013;21:S59-61.

50. National Center for AIDS/STD Control and Prevention. Chinese CDC. In: Ningxia AIDS/HCV sentinel surveliance system 2015 report (Unpublshished). 2015.

51. State Council of P.R. China. China's action plan for reducing and preventing the spread of HIV/AIDS (2006-2010). 2006.

52. May MT, Justice AC, Birnie K, Ingle SM, Smit C, Smith C, Neau D, Guiguet M, Schwarze-Zander C, Moreno S, et al. Injection Drug Use and Hepatitis C as Risk Factors for Mortality in HIV-Infected Individuals: The Antiretroviral Therapy Cohort Collaboration. J Acquir Immune Defic Syndr. 2015;69:348-54.

53. Sidique N, Kohli A, Shivakumar B, Migueles S, Subramanian GM, Naggie S, Polis MA, Masur H, Kottilil S. HIV/HCV-coinfected natural viral suppressors have better virologic responses to PEG-IFN and ribavirin than ARV-treated HIV/HCV patients. J Acquir Immune Defic Syndr. 2011;58:e38-40.

54. Lin ZH, Xin YN, Dong QJ, Wang Q, Jiang XJ, Zhan SH, Sun Y, Xuan SY. Performance of the aspartate aminotransferase-to-platelet ratio index for the staging of hepatitis C-related fibrosis: an updated meta-analysis. Hepatology. 2011;53:726-36

55. Chou R, Wasson N. Blood tests to diagnose fibrosis or cirrhosis in patients with chronic hepatitis $C$ virus infection: a systematic review. Ann Intern Med. 2013:158:807-20

56. Sterling RK, Lissen E, Clumeck N, Sola R, Correa MC, Montaner J, SS M, Torriani FJ, Dieterich DT, Thomas DL. Development of a simple noninvasive index to predict significant fibrosis in patients with HIV/HCV coinfection. Hepatology. 2006:43:1317-25. 